\title{
EFFECTIVENESS OF SHOULDER AND THORACIC MOBILITY EXERCISES ON CHEST EXPANSION AND DYSPNOEA IN MODERATE CHRONIC OBSTRUCTIVE PULMONARY DISEASE PATIENTS
}

\author{
Sonia U Mulay *1, T. Poovishnu Devi ${ }^{2}$, Vaishali Krishnat Jagtap ${ }^{3}$. \\ Krishna College of Physiotherapy, KIMSDU, Karad, India.

\section{ABSTRACT}

Background: Chronic obstructive pulmonary disease (COPD) is characterized by acute exacerbation causing breathlessness, cough and reduced chest expansion. COPD is a currently the fourth leading cause of death in the world. In COPD, expiratory airflow is limited due to obstruction, leading to air trapping and hyperinflation.

Materials and Methods: It was an experimental study carried out at the pulmonology department of Krishna hospital, Karad. A total of 40 subjects were selected by convenient sampling and they were randomly allocated into two groups.Group A received conventional chest physiotherapy and Group B received shoulder and thoracic mobility exercises along with conventional chest physiotherapy. Treatment duration was 30 minutes/session, twice daily for three days. The outcome measures were chest expansion using inch tape and Modified Borg Scale (MBS) for dyspnoea.

Result and Discussion: Paired t test and wilcoxon matched pairs test was used to analyze the effect within the groups. There was significant difference for chest expansion at 3 levels in group A $(p=0.0286),(p=0.0358),(p$ $=0.0298)$ and extremely significant difference in group $B(p<0.0001),(p<0.0001),(p<0.0001)$. In MBS, there was extremely significant difference $(p<0.0001)$ in group $A$ also there was extremely significant difference $(p<0.0001)$ in group $B$.

Unpaired t test and Mann Whitney test was used to analyse the effect between the groups. There was extremely significant difference for chest expansion in post values $(p<0.0001),(p<0.0001),(p<0.0001)$ between group $A$ and $B$. In MBS, post value $(p<0.0001)$ was extremely significant between group $A$ and $B$.

Conclusion: Hence, It was concluded that shoulder and thoracic mobility exercises were more effective in improving chest expansion and reducing dyspnoea in COPD patients.

KEY WORDS: Chronic obstructive pulmonary disease, Breathlessness, Modified Borg scale, Chest expansion, Shoulder and thoracic mobility exercises.

Address for correspondence: Sonia U Mulay, Krishna College of Physiotherapy, KIMSDU, Karad, Satara, Maharashtra, India. E-Mail: mulay.sonia5551@gmail.com

\begin{tabular}{|l|l|}
\hline \multicolumn{3}{|c|}{ Access this Article online } \\
\hline \multirow{2}{*}{ Quick Response code } & International Journal of Physiotherapy and Research \\
& \multicolumn{1}{|c|}{ ISSN 2321- 1822 } \\
www.ijmhr.org/ijpr.html
\end{tabular}

\section{INTRODUCTION}

Chronic obstructive pulmonary disease (COPD) is characterized by chronic airflow limitation of the airways, which can be prevented but cannot be reversed [1]. COPD is a currently the fourth leading cause of death in the world. Prevalence of COPD in 2002 was estimated to be $11.6 / 200$ in men and 8.77/200 in women [2].

COPD is progressive and is characterized acute exacerbation causing breathlessness (dyspnoea), cough and respiratory distress [3]. As COPD worsens, quality of life is affected. COPD is suspected when there is history of exposure to risk factors for the disease along with chronic 
cough, sputum production and/or dyspnoea and that diagnosis must be confirmed with Spirometry. When forced expiratory volume in one second $\left(\mathrm{FEV}_{1}\right)$ divided by forced ventilation capacity (FVC) is $<70 \%$, even after administration of a bronchodilator, the diagnosis is confirmed [4].

In COPD, expiratory airflow is limited due to obstruction, leading to air trapping and hyperinflation. This accentuates when the minute volume or respiratory rate is increased during exercise [5]. The hyperinflation increases strain on the respiratory muscles, which are forced to work in a limited range of movement which is negative pressure/effort relationship, leading to fatigue and increased dyspnoea [5].

Mechanism: In patients with COPD, mechanical efficiency of the respiratory muscle causes decreased rib cage motion and increases abdominal motion leading to paradoxical pattern of breathing [6]. Due to dysfunction of diaphragm, accessory muscles play a major role. The accessory muscle including abdominal muscle weakness and tightness could affect the diaphragm and causes severe breathlessness and poor exercise tolerance level.

Due to mobility exercises, afferent activities of external and internal intercostals muscle spindle are lengthened, to alleviate dyspnoea to minimize the atrophy of respiratory muscle and facilitate coordinated contraction of respiratory muscles. Due to accessory muscle tightness, chest expansion is decreased, where is rib cage movement is also reduced is also reduced which leads to hyperinflation leading to a chronic reduction of the opposition zone of the diaphragm [3].

Lengthening of soft tissues around the chest wall and respiratory muscles helps efficiently in the contraction force and the chest movements by gaining the lung volumes, breathing control .In COPD, muscle length would increase the efficacy of respiratory muscles and promote respiratory mechanics [3].

Accessory muscles are inactive during inspiration at rest in healthy individuals and they act vigorously during physical activity in COPD, ultimately during upper limb, accessory muscles become ineffective in respiration as they perform their major action to sustain the shoulder girdle in position as a consequence, the respiratory work is shifted to the diaphragm which is already at a mechanical disadvantage in patients with COPD [1].

Patients with COPD frequently experience dyspnoea during normal daily activities when they use their upper extremity. During arm exercise, the etiology of the dyspnoea may relate to dynamic hyperinflation, thoracoabdominal dyssynchrony, changes in ventilator and postural muscle recruitment and/or general peripheral muscle reconditioning (related to less use) with decreased upper extremity strength and endurance in the face of increased ventilator and metabolic demands.

Exercise capacity is impaired in COPD, both peak exercise capacity and functional exercise capacity .Besides lung hyperinflation and physical inactivity, ventilation-perfusion mismatch, hypoxemia, cardiovascular problems and muscular changes contribute to reduced exercise capacity [4].

Arm training improves work effort and dynamic hyperinflation and dyspnoea. Furthermore, arm exercise may lead to greater upper extremity exercises and improves exercise capacity, reduced ventilation and oxygen consumption during arm activities.

During arm exercise, the accessory muscles of respiration are required for the arm task and may not be able to contribute breathing. In addition, since the muscles that move that arm and stabilize the trunk are attached to the rib cage, this increases chest wall impedance, which limits the ability to increase tidal volume during activities. It is these impairments in ventilator mechanics which result in termination of arm exercise at low workload for people with COPD compared to healthy subjects [2].

Supported upper limb activities increases the functional residual capacity due to a stretch of thoracic muscles imposed on the rib cage when the arms are raised with weights it strengthens the upper limb muscles and concluded that supported arm activities improves its endurance and quality of life. A supported arm exercise along with coordinated breathing exercises reduces the rate of dyspnoea and improves the 
Sonia U Mulay, T. Poovishnu Devi, Vaishali Krishnat Jagtap. EFFECTIVENESS OF SHOULDER AND THORACIC MOBILITY EXERCISES ON CHEST EXPANSION AND DYSPNOEA IN MODERATE CHRONIC OBSTRUCTIVE PULMONARY DISEASE PATIENTS.Sonia U Mulay, T. POovishnu Devi,

Vaishali Krishnat Jagtap. EFFECTIVENESS OF SHOULDER AND THORACIC MOBILITY EXERCISES ON CHEST EXPANSION AND DYSPNOEA IN

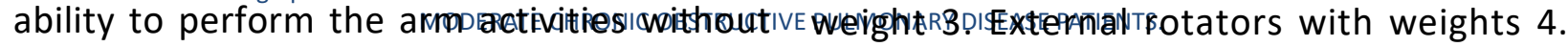
restrictions. Aerobic and strength training in patients with COPD concluded that the combination of strength training and aerobic training showed significant improvement in patients with COPD.

Internal rotators with weight 5. Shoulder shrugged exercise 6 . Shoulder flexion from $0^{\circ}-180^{\circ} 7$. Shoulder extension from $180^{\circ}-0^{\circ}$ 8.Shoulder horizontal abduction 8. Shoulder horizontal adduction.

Upper limb exercise training improves work capacity and endurance and reduces the oxygen consumption during workload. Arm training positively influenced peak arm exercise capacity, metabolic and ventilator demand and also improved activities of daily living.

\section{MATERIALS AND METHODS}

For this study we used Pen, Pencil, Data collection sheet, Inch tape, Dumbbells, Stethoscope.

Study design: Experimental study

Sample design: Convenient sampling with random allocation.

Study setup: Study has carried out on 40 subjects with following inclusion and exclusion criteria 1. Male \& female. 2. Age group between 35- 45yrs. 3. Patient with stage II, Moderate COPD $\mathrm{FEV}_{1} / \mathrm{FVC}<0.70$ according to GOLD classification. 4. Patients who stopped smoking 5 years back. 5 . Patient who are able to comprehend commands. 6 . Ability to give informed consent. Whereas individuals undergone Recent thoracic surgery. Suffering from lung carcinoma, with unstable vital signs, patient having active lung infection. Patient with congenital heart disease, ischemic heart disease, Rheumatic heart disease. Musculo-skeletal disorder that limits the ROM of the joint i.e. arthritis were excluded from the study. Study has conducted at Krishna College of physiotherapy, Karad, Satara, Maharashtra, India.

Outcome measures used for this study are chest expansion measurement with inch tape, Modified Borg Scale for measurement of dyspnoea.

Methodology: Patients were randomly selected based on inclusion and exclusion criteria, they were divided into two groups Control group (Group-A) and Experimental group (Group-B).

Experimental group underwent for 3 days treatment program that included shoulder and thoracic mobility exercises 1 . Shoulder front raise with weight 2 . Shoulder lateral raise with

Dosage:

- Frequency- 2 times/day.

- Treatment duration- 3 days.

- No. of repetitions- 15.

- No of sets- 3

- Total duration- 30 minutes.

Along with that thoracic mobility exercises

\section{Sitting:}

Procedure: The patient should exhale while bending forward to touch the floor with arms crossed at the feet then the patients should extend up while taking a deep inspiration and lift the arm up arm up for 3 days, 3 sets of 10 repetitions.

Standing:

Procedure:The patient should stand with his knees straight, the patient instructed to exhale while bending forward to touch the floor with arms; then the patient should extend up by lifting his hands simultaneously taking a deep inspiration for 3 days, 3 sets of 10 repetitions.

Conventional chest physiotherapy includes:

- Percussion

- Vibration

- Shaking

Breathing exercises:

- Pursed lip breathing

- Diaphragmatic breathing

- Glosso-pharyngeal breathing.

- Deep coughing.

- Forced expiratory technique

Control group was treated with only conventional chest physiotherapy.

Both the groups will be advised to continue their normal activity and follow their inhaler and medications prescribed by the chest physician.

\section{DATA ANALYSIS AND RESULTS CHEST EXPANSION}

Statistical Test: Unpaired $t$ test. Pre interventional analysis showed considered not 
significant difference between Group $A$ and Group B ( $p=0.6993)$.

Statistical Test: Unpaired t test. Pre interventional analysis showed considered not significant difference between Group A and Group B ( $p=0.5177$ ).

Statistical Test: Unpaired t test. Pre interventional analysis showed considered not significant difference between Group A and Group B ( $p=0.4728$ ).

Statistical Test: Unpaired t test. Post interventional analysis showed considered extremely significant difference between Group $A$ and Group B $(p<0.0001)$.

Statistical Test: Paired t test showed considered significant and extremely significant difference for Group A ( $p=0.0286)$ and Group B ( $<<0.0001)$.

Statistical Test: Paired t test showed considered significant and extremely significant difference for Group A ( $p=0.0358)$ and Group B ( $<<0.0001)$.

Statistical Test: Paired t test showed considered significant difference for Group A ( $p=0298)$ and showed extremely significant difference for Group B ( $p<0.0001)$.

\section{MODIFIED BORG SCALE:}

Statistical Test: Mann Whitney test. Pre interventional analysis showed considered not significant difference between Group $A$ and Group B ( $p=0.6909)$.

Statistical Test: Mann Whitney test. Pre interventional analysis showed considered extremely significant difference between Group A and Group B ( $p=<<0.0001)$.

Statistical Test: Wilcox on matched pairs test showed considered extremely significant difference for Group A $(p<0.0001)$ and showed extremely significant difference for Group B $(p<0.0001)$.

\section{DISCUSSION}

40 subjects clinically diagnosed with COPD and fulfilling inclusion and exclusion criteria with age between 35 to 45 years were included in the study. Further they were classified according to GOLD classification as stage 2 (Moderate COPD). They were allocated into two groups, Group $A$ and Group B, each containing 20 subjects. A conventional chest physiotherapy treatment was given in Group A and baseline or conventional chest physiotherapy treatment with an addition to shoulder and thoracic mobility exercises in Group B. The outcome was measured by chest expansion with inch and Modified Borg Scale.

Conventional treatment of chest physiotherapy techniques percussion, vibration, shaking. Breathing exercises include Pursed lip breathing, diaphragmatic breathing, Gloss-pharyngeal breathing, deep coughing; Forced expiratory technique was common for both the groups.

The average mean age of participants in Group

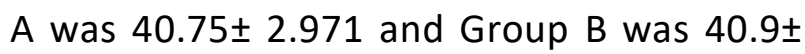
2.972, which showed there is no significant difference in age of subjects in both groups $(t=0.1596 \& p=0.8740)$ ) which was done by unpaired t-test. The total number of participants included over 40 out of which 23 were males and 17 were females. Group A contained 11 males and 9 females and Group B had 12 males and 8 females.

Paired t test and Wilcox on matched pairs test was used to analyze the effect of shoulder and thoracic mobility exercises on chest expansion measurement and dyspnoea within the groups. There was extremely significant and considered significant difference for chest expansion at axillary level, nipple level, xiphisternal level and score of modified Borg scale post 3 days of treatment $(p=0.0286),(p=0.0358),(p=0.0298)$, $(p<0.0001)$ for A group and there was extremely significant and difference for chest expansion at axillary level, nipple level, xiphisternal level and score of modified Borg scale post 3 days of treatment $(p<0.0001),(p<0.0001)$, $(p<0.0001),(p<0.0001)$ for group B.

Unpaired t test and Mann Whitney test was used to analyze the effect of shoulder and thoracic mobility exercises on chest expansion measurement and dyspnoea between the groups. There was considered extremely significant difference for post values of chest expansion at axillary level, nipple level, xiphisternal level $(p<0.0001)$, $(p<0.0001),(p<0.0001)$ and there was considered not significant difference for pre values of chest expansion at axillary level, nipple level, xiphisternal level $(p=0.6993) \quad(p=0.5177)$ $(p=0.4728)$. 
For MBS post $(\mathrm{p}=<0.0001)$ considered extremely significant difference and pre value $(p=0.6909)$ is considered not significant between $A$ and $B$.

The results from the statistical analysis of the present study supported the alternative hypothesis which stated that there will be beneficial effect to the subjects treated with shoulder and thoracic mobility exercises along with conventional chest physiotherapy therapy.

Table 1: Comparison of pre- values of chest expansion at axillary level between the groups.

\begin{tabular}{|c|c|c|}
\hline Group & Mean \pm SD & Median \\
\hline A & $0.7 \pm 0.3120$ & 0.7 \\
\hline B & $0.8 \pm 0.3376$ & 0.8 \\
\hline
\end{tabular}

Table 2: Comparison of pre- values of chest expansion at Nipple level between the groups.

\begin{tabular}{|c|c|c|}
\hline Group & Mean \pm SD & Median \\
\hline A & $0.7 \pm 0.2902$ & 0.7 \\
\hline B & $0.8 \pm 0.2902$ & 0.8 \\
\hline
\end{tabular}

Table 3: Comparison of pre- values of chest expansion at Xiphisternal level between the groups.

\begin{tabular}{|c|c|c|}
\hline Group & Mean \pm SD & Median \\
\hline A & $0.8 \pm 0.1927$ & 0.8 \\
\hline B & $0.7 \pm 0.1997$ & 0.8 \\
\hline
\end{tabular}

Table 4: Comparison of post- values of chest expansion at axillary level between the groups.

\begin{tabular}{|c|c|c|}
\hline Group & Mean \pm SD & Median \\
\hline A & $0.9 \pm 0.3397$ & 0.8 \\
\hline B & $1.3 \pm 0.2536$ & 1.2 \\
\hline
\end{tabular}

Table 5: Comparison of post- values of chest expansion at nipple level between the groups.

\begin{tabular}{|c|c|c|}
\hline Group & Mean \pm SD & Median \\
\hline A & $0.9 \pm 0.3528$ & 0.8 \\
\hline B & $1.3 \pm 0.2501$ & 1.3 \\
\hline
\end{tabular}

Table 6: Comparison of post- values of chest expansion at xiphisternal level between the groups.

\begin{tabular}{|c|c|c|}
\hline Group & Mean \pm SD & Median \\
\hline A & $0.8 \pm 0.1875$ & 0.9 \\
\hline B & $1.1 \pm 0.1905$ & 1.1 \\
\hline
\end{tabular}

Table 7: Comparison of pre-values and post- values of chest expansion at axillary level within the groups.

\begin{tabular}{|c|c|c|}
\hline \multirow{2}{*}{ Group } & \multicolumn{2}{|c|}{ Median } \\
\cline { 2 - 3 } & Pre & Post \\
\hline A & 0.7 & 0.8 \\
\hline B & 0.8 & 1.2 \\
\hline
\end{tabular}

Table 8: Comparison of pre-values and post- values of chest expansion at nipple level within the groups.

\begin{tabular}{|c|c|c|}
\hline \multirow{2}{*}{ Group } & \multicolumn{2}{|c|}{ Median } \\
\cline { 2 - 3 } & Pre & Post \\
\hline A & 0.8 & 0.8 \\
\hline B & 0.8 & 1.3 \\
\hline
\end{tabular}

Table 9: Comparison of pre-values and post- values of chest expansion at xiphisternal level within the groups.

\begin{tabular}{|c|c|c|}
\hline \multirow{2}{*}{ Group } & \multicolumn{2}{|c|}{ Median } \\
\cline { 2 - 3 } & Pre & Post \\
\hline A & 0.8 & 0.9 \\
\hline B & 0.7 & 1.5 \\
\hline
\end{tabular}

\section{MODIFIED BORG SCALE:}

Table 10: Comparison of pre- values of Modified Borg scale between the groups.

\begin{tabular}{|c|c|c|}
\hline Group & Mean \pm SD & Median \\
\hline A & $4.6 \pm 0.8208$ & 4.5 \\
\hline B & $4.4 \pm 0.6048$ & 2 \\
\hline
\end{tabular}

Table 11: Comparison of post- values of Modified Borg scale between the groups.

\begin{tabular}{|c|c|c|}
\hline Group & Mean \pm SD & Median \\
\hline A & $3.5 \pm 0.8256$ & 3 \\
\hline B & $2.2 \pm 0.6387$ & 2 \\
\hline
\end{tabular}

Table 12: Comparison of pre-values and post- values of Modified Borg Scale within the groups.

\begin{tabular}{|c|c|c|}
\hline \multirow{2}{*}{ Group } & \multicolumn{2}{|c|}{ Median } \\
\cline { 2 - 3 } & Pre & Post \\
\hline A & 4.5 & 3 \\
\hline B & 4.5 & 2 \\
\hline
\end{tabular}

\section{CONCLUSION}

Chronic obstructive pulmonary disease (COPD) is characterized by chronic airflow limitation of the airways, which can be prevented but cannot be reversed [1]. COPD is progressive and is characterized acute exacerbation causing causing breathlessness (dyspnoea), cough and respiratory distress [3].

Dyspnoea is the most debilitating condition aspects of COPD that leads to disability and poor quality of life resulting in frequent access to rehabilitation. COPD is a currently the fourth leading cause of death in the world. Prevalence of COPD in 2002 was estimated to be 11.6/1000 in men and 8.77/1000 in women.

So the present clinical trial was conducted to find out the effect of shoulder and thoracic mobility exercises on chest expansion and dyspnoea in moderate COPD patients.

40 subjects clinically diagnosed with COPD and fulfilling inclusion and exclusion criteria with age between 35 to 45 years were included in the study. Further they were classified according to GOLD classification as stage 2 (Moderate COPD). They were allocated into two groups, 
Group A and Group B, each containing 20 subjects. A conventional chest physiotherapy treatment was given in Group $A$ and baseline or conventional chest physiotherapy treatment with an addition to shoulder and thoracic mobility exercises in Group B. The outcome was measured by chest expansion with inch and Modified Borg Scale.

Conventional treatment of Chest physiotherapy techniques Percussion, Vibration, Shaking. Breathing exercises include Pursed lip breathing, diaphragmatic breathing, Glosspharyngeal breathing, deep coughing; Forced expiratory technique was common for both the groups.

The average mean age of participants in Group A was $40.75 \pm 2.971$ and Group B was 40.9 \pm 2.972 , which showed there is no significant difference in age of subjects in both groups $(t=0.1596 \& p=0.8740)$ ) which was done by unpaired t-test. The total number of participants included over 40 out of which 23 were males and 17 were females. Group A contained 11 males and 9 females and Group B had 12 males and 8 females.

Paired $t$ test and wilcoxon matched pairs test was used to analyse the effect of shoulder and thoracic mobility exercises on chest expansion measurement and dyspnoea within the groups. There was extremely significant and considered significant difference for chest expansion at axillary level, nipple level, xiphisternal level and score of modified Borg scale post 3 days of treatment $(p=0.0286),(p=0.0358),(p=0.0298),(p<$ 0.0001 ) for A group and there was extremely significant and difference for chest expansion at axillary level, nipple level, xiphisternal level and score of modified Borg scale post 3 days of treatment $(p<0.0001),(p<0.0001),(p<$ $0.0001),(p<0.0001)$ for group $B$

Unpaired t test and Mann Whitney test was used to analyse the effect of shoulder and thoracic mobility exercises on chest expansion measurement and dyspnoea between the groups. There was considered extremely significant difference for post values of chest expansion at axillary level, nipple level, xiphisternal level $(p<0.0001),(p<0.0001)$, $(p<0.0001)$ and there was considered not significant difference for pre values of chest expansion at axillary level, nipple level, xiphisternal level $(p=0.6993)(p=0.5177)(p=0.4728)$. For MBS post $(p=<0.0001)$ considered extremely significant difference and pre value $(p=0.6909)$ is considered not significant between $A$ and $B$.

The results from the statistical analysis of the present study supported the alternative hypothesis which stated that there will be beneficial effect to the subjects treated with shoulder and thoracic mobility exercises along with conventional chest physiotherapy therapy.

Hence above results showed that Group B subjects treated with shoulder and thoracic mobility exercises along with conventional therapy showed better improvement in chest expansion and reduction of dyspnoea.

Thus it can be stated from above study that physical therapy interventions like shoulder and thoracic mobility exercises are more efficacious and cost effective.

\section{ABBREVATIONS}

COPD - Chronic Obstructive Pulmonary Disease MBS - Modified Borg scale.

\section{Conflicts of interest: None}

\section{REFERENCES}

[1]. K.Rekha,Vijayalakshmi A,D Samuel Sundar Doss ,Vaiyapuri Anandha. Effect of home based upper extremity exercises in chronic obstructive pulmonary disease. Clinical Research 2016;8(9):1351-1355

[2]. Ganesan Kathiresan, Senthil Kumar Jeyaram , Jayachandra Jaganathan. Effect of upper extremity exercises in people with COPD. J Thorac Dis 200. 2ISSN: 2072-1439.

[3]. Rekha K,Shristi Rai,Vaiyapuri Anandh,Samuel Sundar Doss D. Effect of stretching respiratory accessory muscles in chronic obstructive pulmonary disease. ASIAN JORNAL OF PHARMACEUTICALS ANS CLINICAL RESEARCH 2016;9(1);2455-3891.

[4]. Parmar Dharmesh Journal of exercise sciences and physiotherapy published by Exercise Fitness and Health Alliance JESP 2015;11(2):134-141. DOI: $10.18376 / 2015 / v 11 \mathrm{i} / 67713$.

[5]. Bellemare, F Grassino,A. Force reverse of the diaphragm in patients with COPD.J Appl.Physiol., 1983;55:8-15.

[6]. Ito M, Kakizaki F, Tsuzura Y,Yamada M. Immediate effect of respiratory muscle stretch gymnastic and diaphragmatic on respiratory pattern. Respiratory muscle Conditioning Group. Intern Med 1999;38(2):126-32.

[7]. Lisa Hopp PhD, RN, Jane Walker, PhD, RN. Effectiveness of Arm Exercise on Dyspnea in patients with Chronic Obstructive Pulmonary Disease: A Systemic Review. 